\title{
Opposite effects of dexamethasone and retinoic acid on neuronal actin cytoskeleton
}

Hélène Roumes ${ }^{1,2}$, Julie Brossaud $^{1,2}$, Aloïs Lemelletier ${ }^{1}$, Jean-Christophe Helbling1, Marie-Pierre Moisan ${ }^{1}$, Véronique Pallet $^{1,2}$, Anabelle Redonnet ${ }^{1,2}$, Jean-Benoît Corcuff ${ }^{1,2}$

1 INRA, Nutrition et Neurobiologie Intégrée, UMR1286, F-33076 Bordeaux ;2 Université de Bordeaux, Nutrition et Neurobiologie Intégrée, UMR 1286 , F-33076 Bordeaux, France

\section{Introduction and objectives}

Retinoic acid (RA) implication is crucial in adult brain through its involvement in cellular and synaptic plasticity process [1]. By contrast, glucocorticoids (GC) impair synaptic plasticity [2]. We questioned the influence of RA and Dex on hippocampal HT-22 cell line morphology via the modulation of F-actin cytoskeleton organization and the implication of calpain system.
Methods: HT22 hippocampal murine cells were grown 4d with vehicule, RA, Dex or RA/Dex at $10^{-6} \mathrm{M}$. After treatment with or without calpeptin, the cells were fixed and their morphologies were observed in phase contrast. F-actin was stained with phalloidin-FITC $(0.5 \mu \mathrm{M})$ and nucleus with DAPI $(1.5 \mu \mathrm{g} / \mathrm{ml})$ for $40 \mathrm{~min}$. The cells were observed using an epifluorescence microscope. Calpain activity was quantified in vitro with the Abcam kit. p44/42 MAPK (ERK1/2) antibody were provided by Cell Signaling Biotechnology

\section{Results}

1 RA and Dex have opposite effect on cell morphology modifying F-actin and stress fibres of the cytoskeleton
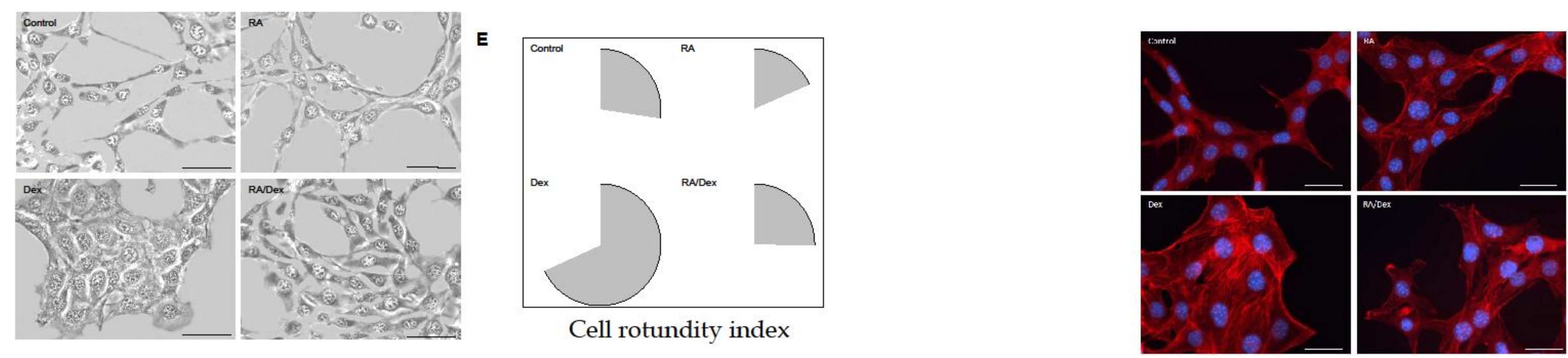

$\underline{\text { RA and Dex effects on stress fibres but not F-actin is linked with the modification of calpain activity }}$
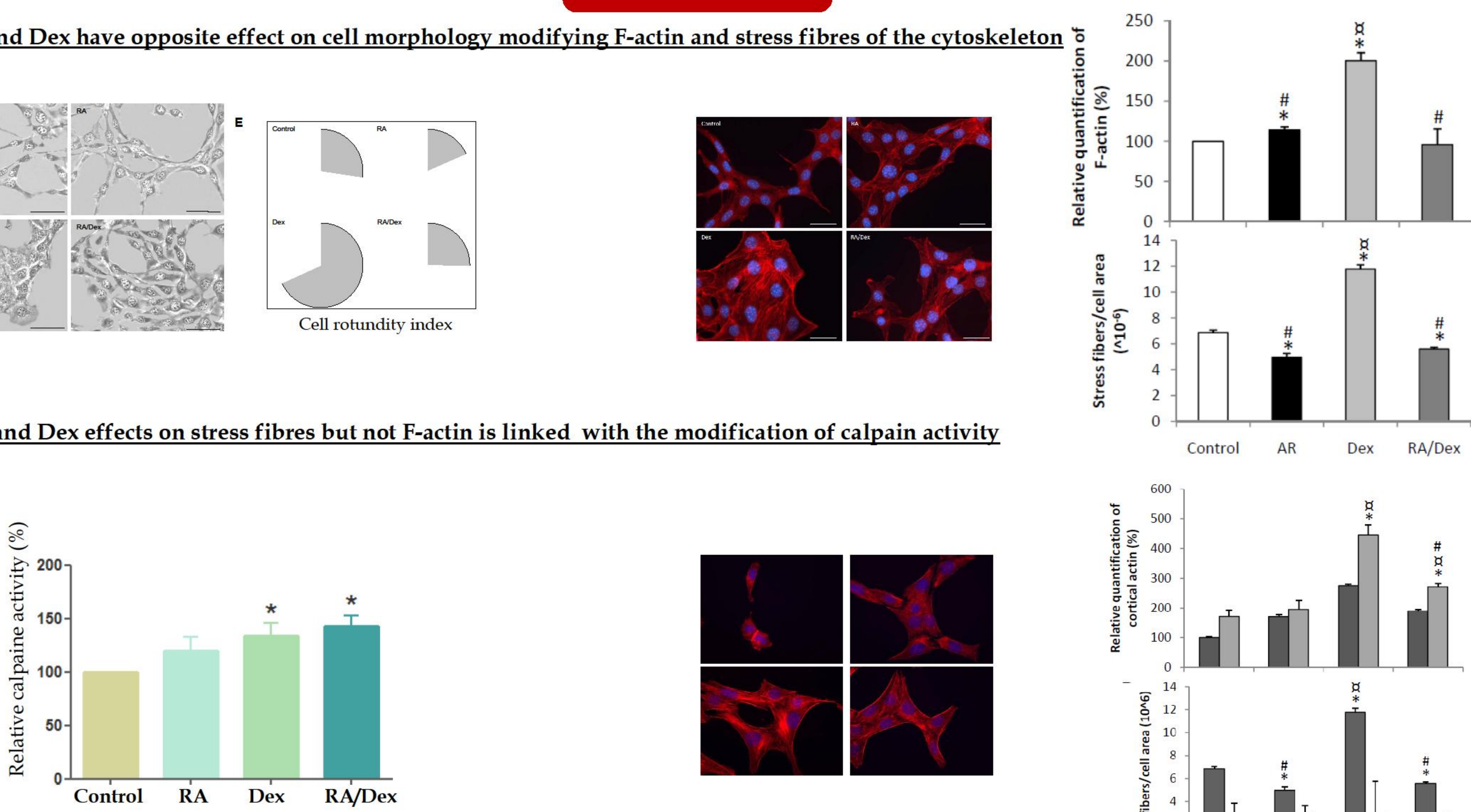

3

$\underline{\text { RA and Dex interact on phospho- and total- MAPK p42/44 }}$

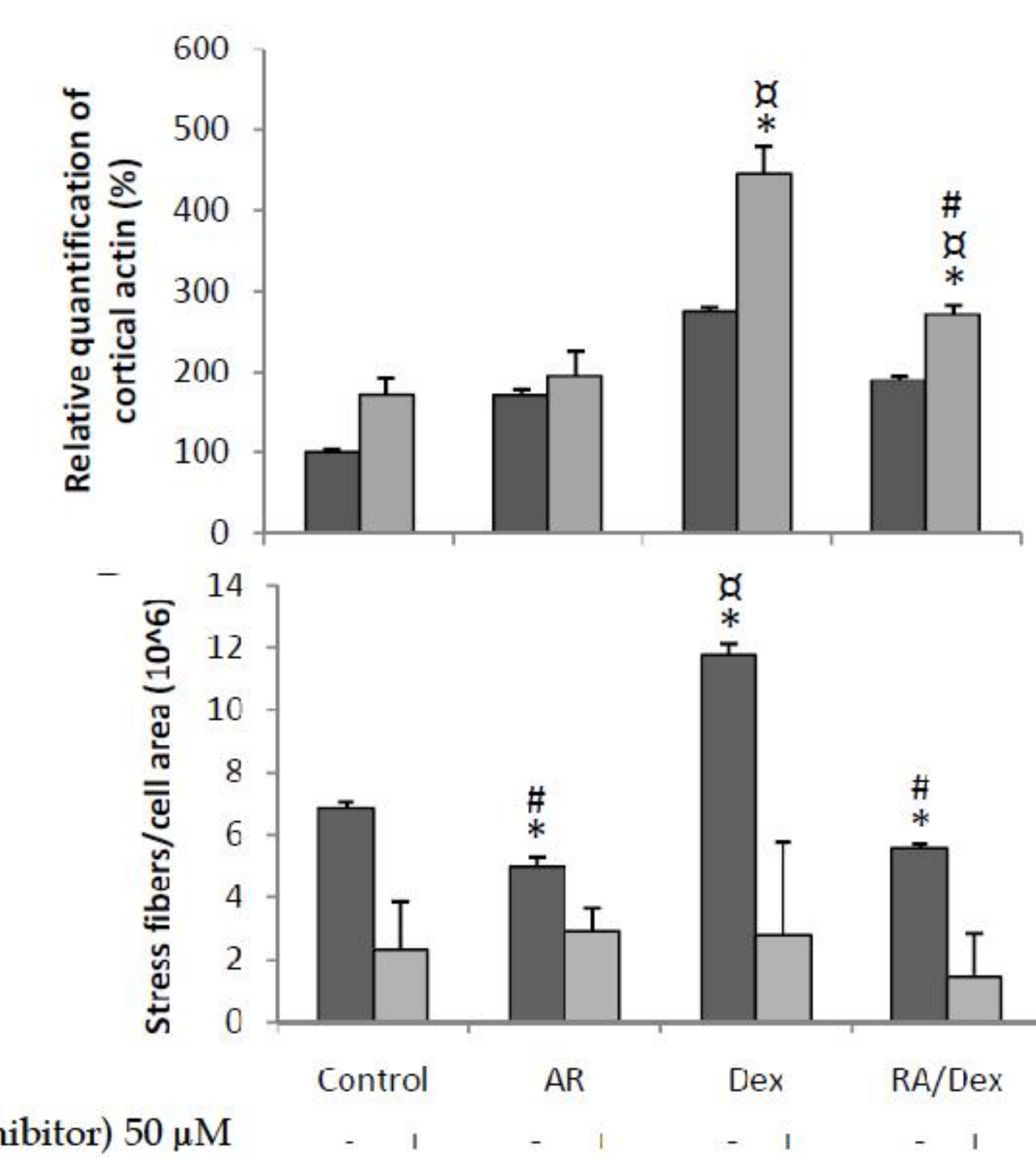

Control RA Dex $\quad$ RA/Dex
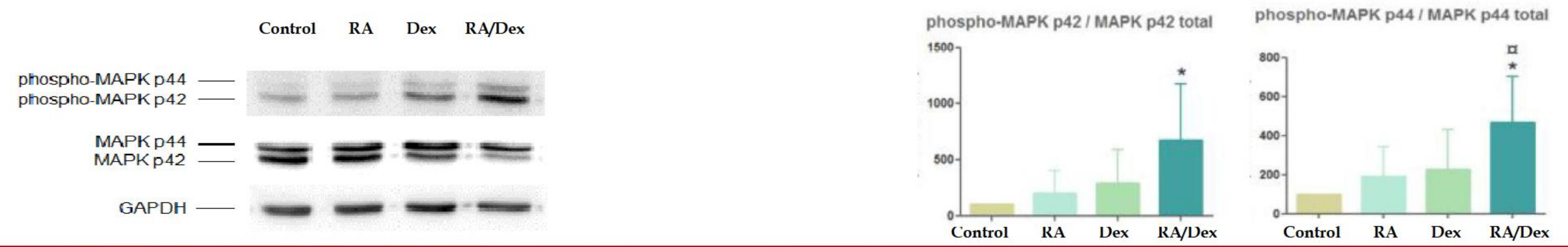

\section{Conclusion}

From these data, we propose the following mechanism:

RA and Dex modify calpain activity via a modification of the ERK/MAPK pathway. This could explain RA and Dex effects on stress fibres because of the implication of calpain activity in the polymerisation of the cytoskeleton

References: 1-Lane, M.A. and S.J. Bailey, Role of retinoid signalling in the adult brain. Prog Neurobiol, 2005 2-Alfarez, D.N., et al., Corticosterone and stress reduce synaptic potentiation in mouse hippocampal slices with mild stimulation. Neuroscience, 2002

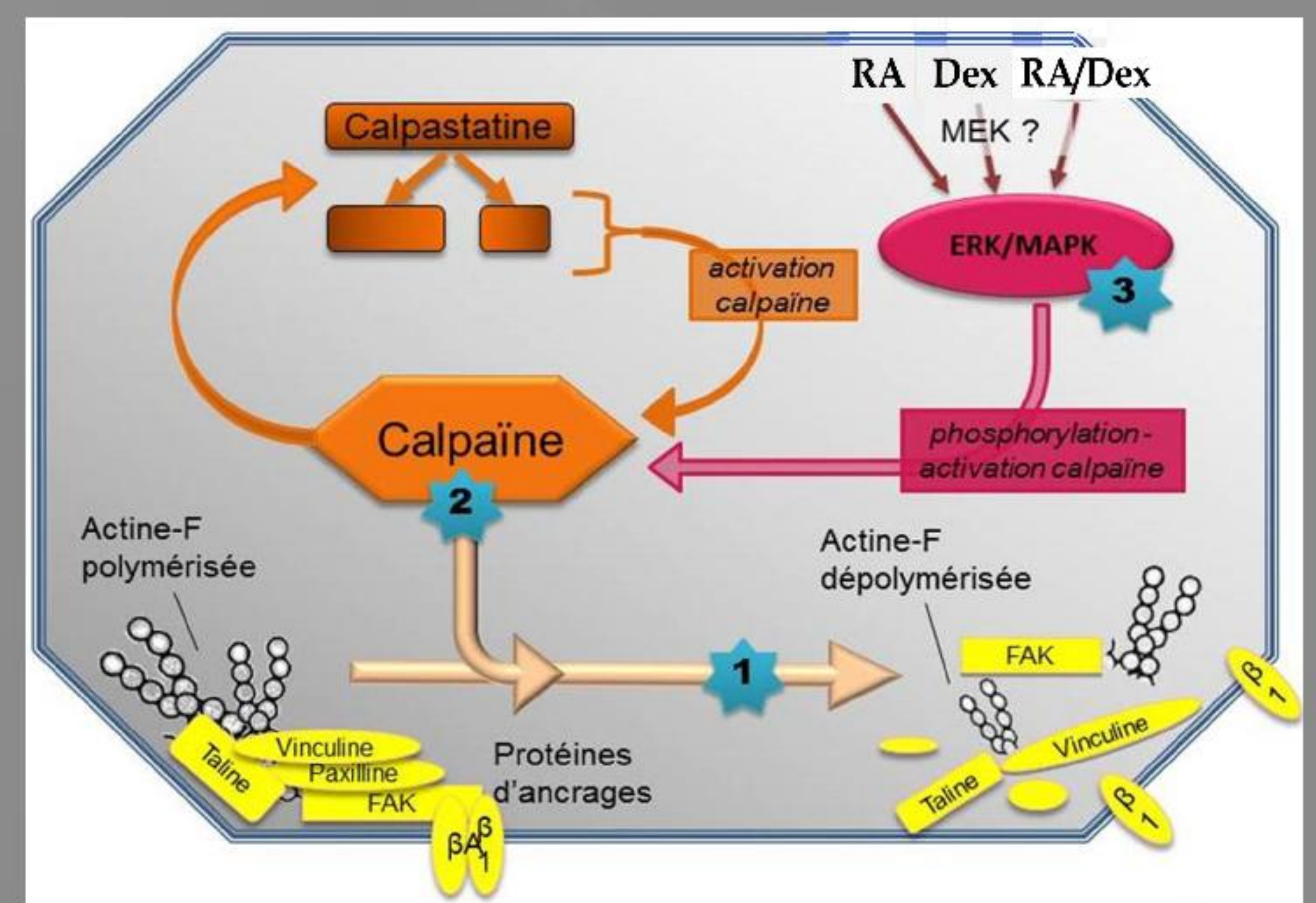

